\title{
ON THE NATURE OF WEIGHTED ESSENTIALLY NONOSCILLATORY SCHEMES IN SHOCKED FLOWS
}

\author{
E. S. FERREIRA JR ${ }^{1}$, R. B. DE R. BORGES ${ }^{2}$ AND S. S. V. VIANNA ${ }^{1}$ \\ ${ }^{1}$ University of Campinas, School of Chemical Engineering \\ ${ }^{2}$ State University of Rio de Janeiro, Department of Mathematics \\ Email para contato: elmo@feq.unicamp.br / rafael.borges@ime.uerj.br / \\ savio@feq.unicamp.br
}

\begin{abstract}
Nozzle flows have many applications in engineering. Therefore, numerical improvements in such problems are always relevant. Following these lines, we investigated how the WENO (Weighted Essentially Nonoscillatory) scheme deals with shocked nozzle flows. The inviscid flow is modelled via the Euler equations and the numerical findings are compared with the classical MacCormack scheme. Results show good agreement between the compared solutions.
\end{abstract}

\section{INTRODUCTION}

Shock wave propagation and contact discontinuities are common physical phenomena that occur in a nozzle flow. Traditional numerical techniques may employ artificial viscosity to deal with shocked nozzle flows in order to avoid numerical oscillations near shocks. On the other hand, the WENO (Weighted Essentially Nonoscillatory) scheme adapts itself to the discontinuity caused by the shock by evaluating whether the region is smooth or not (Jiang e Shu, 1996). This work aims to investigate the potential application of the WENO scheme in nozzle flow problems, including a procedure to solve the inviscid Euler equations in a quasi-one-dimensional form by the WENO scheme. The numerical findings are compared with the MacCormak technique and the exact solution of the problem under investigation. Analysis of the results shows good agreement.

\section{NOZZLE FLOW MODELLING}

The governing equations for the nozzle flow discussed in this work comprise the quasione-dimensional or unsteady Euler equation of the flow and a source term that considers the area variation along the nozzle distance. It can be described by equation 1 (Jiang e Shu, 1996).

$$
\left[\begin{array}{l}
\rho \\
\rho u \\
E
\end{array}\right]_{t}+\left[\begin{array}{l}
\rho u \\
\rho u^{2}+p \\
u(E+p)
\end{array}\right]_{x}=-\frac{A_{x}}{A}\left[\begin{array}{l}
\rho u \\
\rho u^{2} \\
u(E+p)
\end{array}\right] .
$$

The first vector storages the conserved variables while the second vector considers the fluxes of mass, momentum and energy. The variables $\rho, u, P$ and $E$ are the density, velocity, pressure, and total energy. The cross area of the nozzle is $A=A(x)$ and its derivative is $A_{x}=\frac{d A}{d x}$. 


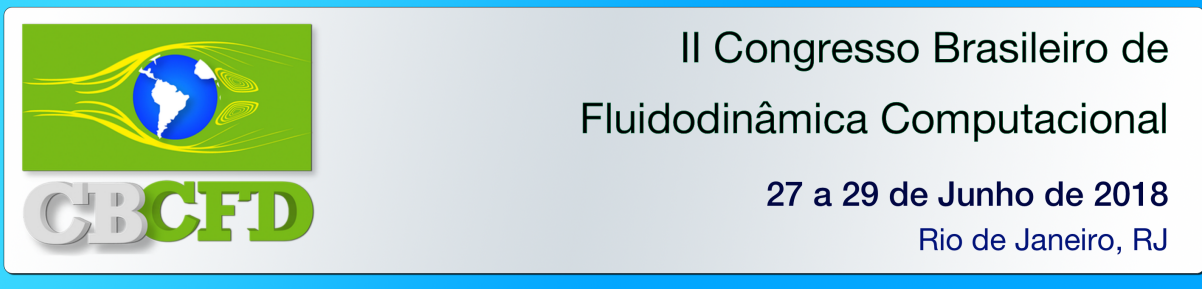

Equation 1 was solved by the fifth-order WENOZ scheme (Borges et al., 2008). The solution procedure is the same as computing regular Euler except for the source term and the flux derivative. The flux of mass $(\rho u)$, momentum $\left(\rho u^{2}+p\right)$ and energy $(u(E+p))$ was calculated and reconstructed. The flux of mass and energy in the source term followed the same approach scaled by the negative ratio of area derivative and the cross area of the nozzle $\left(\frac{-A_{x}}{A}\right)$. The reconstructed momentum flux $\left(\rho u^{2}+p\right)$, however, must be subtracted from the pressure $(p)$ in order to obtain the corresponding momentum source term $\left(\rho u^{2}\right)$. The simplified Fortran instructions below clarifies how the procedure was implemented in the developed code. The source terms for mass, momentum and energy are, respectively, phi $(1, i), \operatorname{ph} i(2, i)$ and $\operatorname{ph} i(3, i)$, whereas, variables flux $(1, i), f l u x(2, i)$ and $f l u x(3, i)$ are the WENOZ reconstructed fluxes of mass, momentum and energy, respectively.

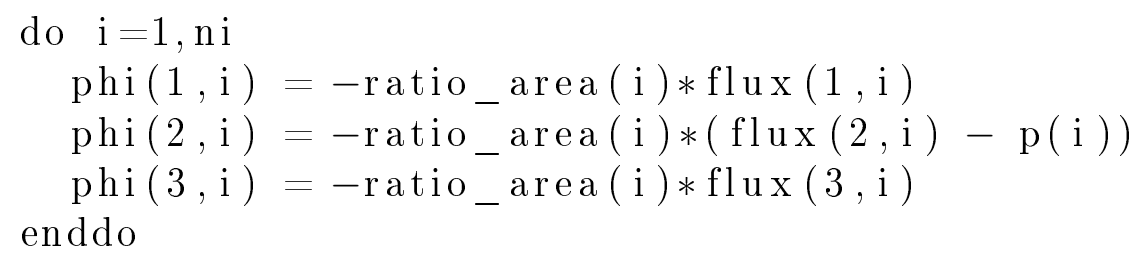

The compact form of equation 1 can be written as

$$
Q_{t}+F_{x}=\phi
$$

where $Q, F$ and $\phi$ are conserved variables, the fluxes and the source term, respectively. In a semi-descretised form the equation 2 above becomes

$$
Q_{t}=\frac{F_{i+1}-F_{i}}{\Delta x}+\phi_{i+1 / 2}
$$

The conserved variables, $Q_{t}$, advance in time based on TVD-Runge-Kutta as reported by (Gottlieb e Shu, 1998). The current work used the TVD second-order Runge-Kutta method. All equation and numerical schemes are implemented in Fortran 90.

\section{NOZZLE CASE}

The shocked nozzle flow problem is presented in Figure 1 where the boundaries contours are described in accordance with the parabolic behaviour given by,

$$
A=1+2.2(x-1.5)^{2} \quad 0 \leq x \leq 3.0 .
$$

The throat of the nozzle is at $x=1.5$, the convergent section is $x<1.5$ while the divergent section is $x>1.5$. Figure 1 shows the schematic of the nozzle flow with internal normal shock wave. The nondimensinal boundary condition at point 1 was considered as a fixed value for density $\left(\rho_{1}=1\right)$ and temperature $\left(T_{1}=1\right)$. The pressure $\left(p_{1}=\rho_{1} T_{1}\right)$ was calculated by the ideal gas equation. The velocity was computed based on the internal points by linear extrapolation $\left(V_{1}=2 V_{2}-V_{3}\right)$. In the boundary condition at point $N$ all variables were computed based on the internal points, except the pressure which remains with a fixed value $\left(p_{N}=0.6784 p_{1}\right)$. The outlet temperature was computed by linear extrapolation while the density by the ideal gas equation. 

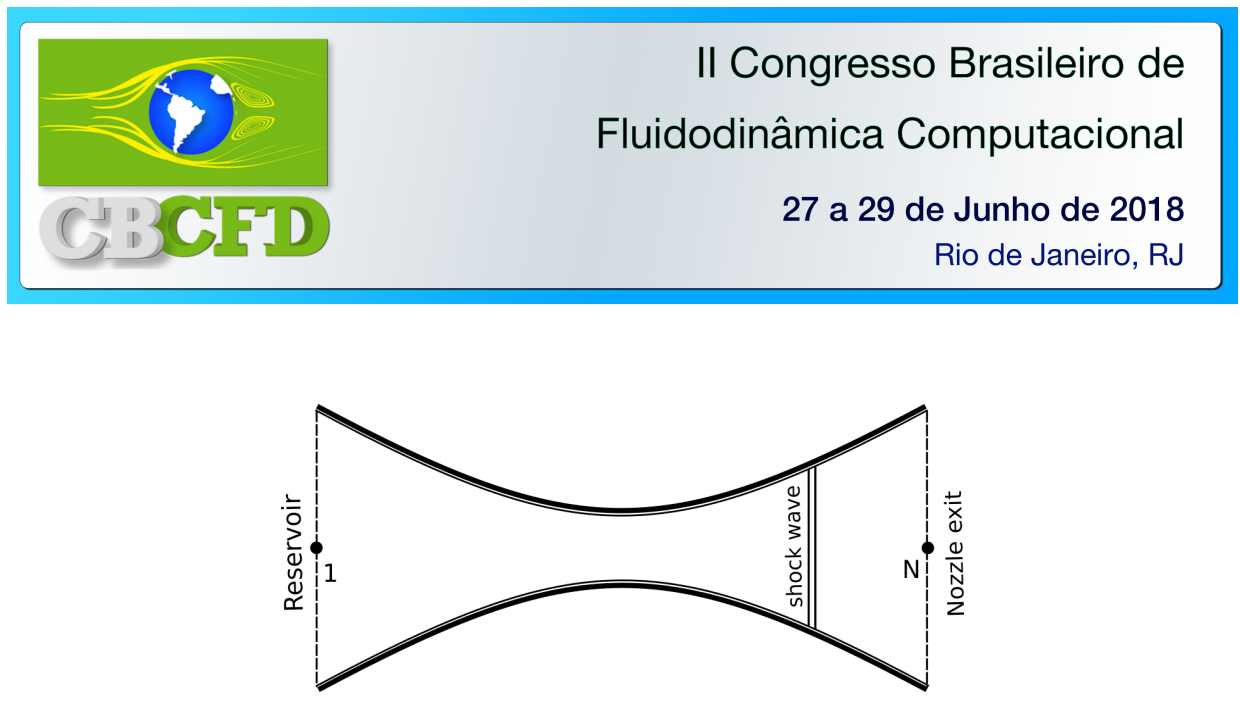

Figure 1 - Schematic of a nozzle flow with an internal normal shock wave. Node 1 is the inlet flow while node $N$ is the outlet flow.

\section{RESULTS}

The normal shock wave appears in the flow field and WENOZ and MacCormark schemes solution have been considered during the modelling of the case. Table 1 shows the values of $\rho / \rho_{0}, p / p_{0}$ and $M$ at the nozzle throat computed by the exact forms, WENOZ, and MacCormark using artificial viscosity of $a d=0.1$, ad $=0.2$ and $a d=0.3$. The WENOZ scheme achieved good results while MacCormak solved with $a d=0$ fail to capture the physics of the problem. When the artificial viscosity is set to $a d=0.2$, the findings are better than $a d=0.1$ and $a d=0.3$. Finding the best value for the artificial viscosity is quite always challenging as most of the practical problems in fluid mechanics does not hold an analytical solution. Additionally, the utilisation of smooth techniques also introduces new sources of errors. If we know nothing about the exact results presented in Table 1, from nozzle flow theory we could assume that in the nozzle throat the Mach number must be equal to 1 . Thus, observing the Mach number results the minor error is obtained for $a d=0.3$. However, we do know the exact value, and $a d=0.3$ presents worse results than those presented by $a d=0.1$ as far as the density and the pressure are considered. Furthermore, good values for artificial viscosity that are verified in a specific point (throat) of the flow may not be good for all the flow field. Figure 2 shows the non-dimensional pressure behaviour in a shocked flow with a shock in a divergent zone. The shock was well captured by the WENOZ formulation. For the MacCormack scheme, wiggling trends are noticed for $a d=0$. A more stable solution is reported for $a d=0.2$, although small oscillations are presented at the end of the shock.

\section{CLOSING REMARKS}

The WENOZ scheme emerges as a potential numerical alternative to deal with discontinuity in engineering flows. The scheme has captured the shock showing good agreement with analytical solution without the burden of undesirable oscillations. Further investigation must be touched upon the nature of elapsed computational time. 


\section{Congresso Brasileiro de}

Fluidodinâmica Computacional

27 a 29 de Junho de 2018

Rio de Janeiro, RJ

Table 1 - Value at the nozzle throat for exact solution, WENOZ scheme and MacCormark technique with artificial viscosity of $a d=0.1, a d=0.2$ and $a d=0.3$.

\begin{tabular}{|llll|}
\hline & $\frac{\rho}{\rho_{0}}$ & $\frac{p}{p_{0}}$ & $M$ \\
\hline Exact solution & 0.634 & 0.528 & 1.000 \\
\hline WENO & 0.632 & 0.525 & 1.014 \\
\hline MacCormark $(a d=0.1)$ & 0.629 & 0.523 & 1.016 \\
MacCormark $(a d=0.2)$ & 0.633 & 0.527 & 1.009 \\
MacCormark $(a d=0.3)$ & 0.640 & 0.535 & 0.997 \\
\hline
\end{tabular}
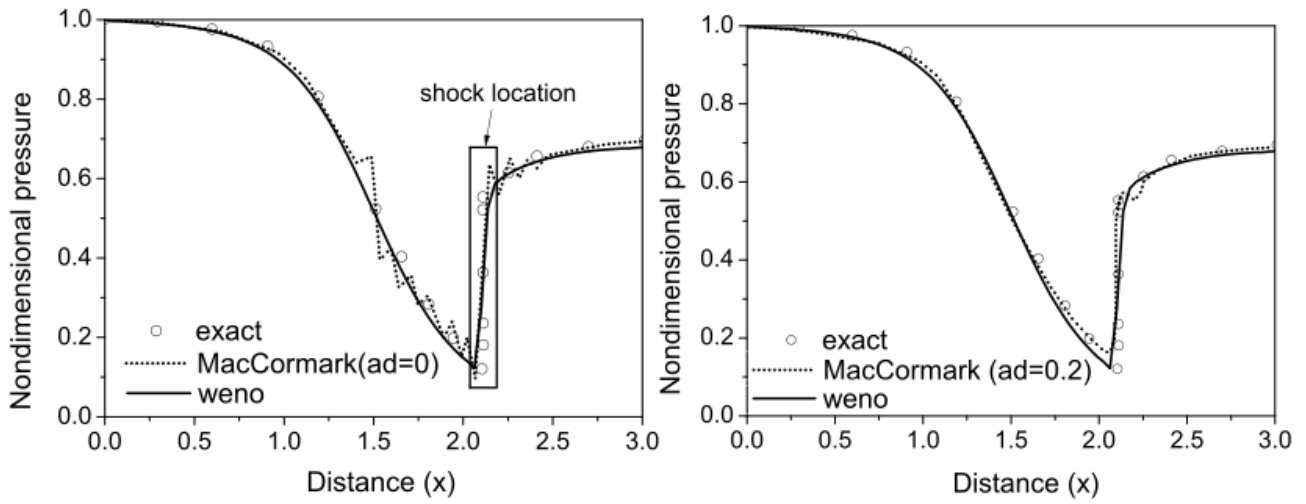

Figure 2 - Shock-capturing numerical results using MacCormark with ad $=0.0$ and $a d=0.2$, and WENOZ for the non-dimensional pressure distribution through the nozzle. Exact solution (circles).

\section{ACKNOWLEGMENTS}

Thanks are due to CAPES, UNICAMP and L4R1S4 laboratory. Support form CENAPAD$\mathrm{SP}$ is much appreciated.

\section{REFERENCES}

Borges, R.; Carmona, M.; Costa, B.; Don, W. S. An Improved Weighted Essentially Non-oscillatory Scheme for Hyperbolic Conservation Laws. Journal Computational Physics, 227, 3191-3211, 2008.

Gottlieb, A.; Shu, C. Total Variation Diminishing Runge-kutta Schemes. Math. Comp., 67, 73-85, 1998.

JiAng, G. S.; Shu, C. W. Efficient implementation of weighted ENO schemes. J. of Comp. Physics, 126, 202-228, 1996. 